$1-1994$

\title{
Oscillating Flow in Channels with Sudden Change in Cross Section
}

Mounir B. Ibrahim

Cleveland State University, m.ibrahim@csuohio.edu

Waqar Hashim

Cleveland State University

Follow this and additional works at: https://engagedscholarship.csuohio.edu/enme_facpub

Part of the Mechanical Engineering Commons

How does access to this work benefit you? Let us know!

\section{Original Citation}

Ibrahim, M., and Hashim, W., 1994, "Oscillating Flow in Channels with a Sudden Change in Cross Section," Computers \& Fluids, 23(1) pp. 211-224.

This Article is brought to you for free and open access by the Mechanical Engineering Department at EngagedScholarship@CSU. It has been accepted for inclusion in Mechanical Engineering Faculty Publications by an authorized administrator of EngagedScholarship@CSU. For more information, please contact library.es@csuohio.edu. 


\title{
OSCILLATING FLOW IN CHANNELS WITH A SUDDEN CHANGE IN CROSS SECTION
}

\author{
MOUNIR Ibrahim and WaQAR HaShIM $\dagger$ \\ Mechanical Enginering Department, Cleveland State University, Euclid Avenue at East 24th Street, \\ Cleveland, OH 44115, U.S.A.
}

\begin{abstract}
In this paper, we have computationally examined oscillating flow (zero mean) between two parallel plates with a sudden change in cross section. The flow was assumed to be laminar incompressible with the inflow velocity uniform over the channel cross section but varying sinusoidally with time. The cases studied cover wide ranges of $\mathbf{R e}_{\max }$ (from 187.5 to 2000), $\mathrm{Va}$ (from 1 to 10.66), the expansion ratio (1:2 and 1:4) and $A_{\mathrm{r}}$ (2 and 4). Also, three different geometric cases were discussed: (a) asymmetric expansion/contraction; (b) symmetric expansion/contraction; and (c) symmetric blunt body. For these oscillating flow conditions, the fluid undergoes sudden expansion in one-half of the cycle and sudden contraction in the other half. The instantaneous friction factor, for some ranges of $\mathbf{R e}_{\max }$ and $\mathrm{Va}$, deviated substantially from the steady-state friction factor for the same flow parameters. A region has been identified (see Fig. 3) below which the flow is laminar quasi-steady. A videotape showing computer simulations of the oscillating flow demonstrates the usefulness of the current analyses in providing information on the transient hydraulic phenomena.
\end{abstract}

\section{NOMENCLATURE}

$A_{\mathrm{r}}-$ Relative amplitude of the fluid displacement [see equation (11)]

$D_{\mathrm{h}}$-Hydraulic diameter of the smaller channel

$f$-Instantaneous friction factor $\left(=2 \tau_{\mathrm{w}} / \rho U_{\mathrm{m}}^{2}\right)$

$h$-Height of the smaller channel

$H-$ Height of the larger channel

L-Total channel length

$P$-Pressure

Re-Instantaneous Reynolds number

$S$-Step size (see Fig. 1)

St-Strouhal number [see equation (12)]

$t$-Time

$T$-Time period for one cycle

$U$-X-component of velocity

$V-Y$-component of velocity

$\mathrm{Va}-$ Valensi number [see equation (10)]
$X$-Distance along the channel axis

$Y$-Distance normal to the channel axis

Greek symbols

$\rho$-Density of the fluid

$\mu$-Dynamic viscosity of the fluid

$\omega$-Frequency of oscillation

\section{Subscripts}

$\mathrm{Dh}-$ Based on the hydraulic diameter

$\mathrm{i}$-Based on the inlet condition

$m$-Mean value

max-Maximum during the cycle

min-Minimum during the cycle

0 - Initial condition/reference state

ss-Steady state

w-At the wall

\section{INTRODUCTION}

Several engineering applications encounter unsteady flow as well as sudden changes in the channel cross section. In free-piston Stirling engine applications, the flow oscillates around a zero mean while sudden changes in the cross section take place at the components interface. As an example, in the NASA SPRE (Space Power Research Engine) the flow goes through a sudden change in cross section at the interface between the heater and the expansion space as well as between the cooler and the compression space. Today, a steady-state correlation for the fluid flow and heat transfer are used in the design analyses of such engines.

Typically, under these oscillating flow conditions the fluid undergoes a sudden expansion in one-half of the cycle and a sudden contraction in the other half. The flow reversal is caused not only by the flow oscillation but also by the sudden expansion.

Since the flow in Stirling engines oscillates in a cyclic manner, the velocity and temperature profiles differ significantly from those obtained for steady flows [1-3]. Accordingly, the friction 
factor and heat transfer coefficient are considerably different from those of the steady-state correlations.

Several computational, experimental and analytical efforts have been conducted to examine the oscillating flows within a straight channel (circular pipe and parallel plates); Ibrahim et al. [1] and Kurzweg [2] have performed several numerical investigations. Simon and Seume $[3,4]$ conducted an experimental analysis for oscillating flow in a circular pipe. Their results show a significant increase in the friction factor as compared to steady flow conditions.

A literature survey shows that several investigations have been conducted for flows with a sudden change in the area of the cross-section. Examples involve steady unidirectional flow over a backward-facing step [5-8] and flow through a sudden contraction in a channel [9-11]. The results obtained showed an enhancement in the friction factor as compared to a straight geometry under similar flow conditions.

In this paper, results from a computational analysis of the flow between two parallel plates with a sudden change in cross section are presented. The flow parameters are selected to emulate the NASA SPRE. Several geometries and expansion ratios have been examined to identify the flow characteristics under different engine operating conditions.

Since experimental data for such a problem do not exist, a careful step-by-step procedure has been used for validating the computer code. This includes, examination of false diffusion, comparison with available data for steady flows as well as solving for impulsively started flows.

\section{ANALYSIS}

\section{Assumptions}

Figure 1 shows the channel with two parallel plates and a sudden change in cross section and the Cartesian coordinate system used for the present analyses. Different geometries are considered: (a) asymmetric expansion/contraction [Fig. 1(a)]; (b) symmetric expansion/ contraction [Fig. 1(b)]; and (c) a symmetric blunt body [Fig. 1(c)]. The following assumptions were made: (1) the flow is laminar incompressible with constant thermophysical properties; (2) the inlet velocity is uniform but varies sinusoidally with time; and (3) the location of the step is far away from both ends; this is chosen to isolate the effect of the step on the flow field from the end effects.

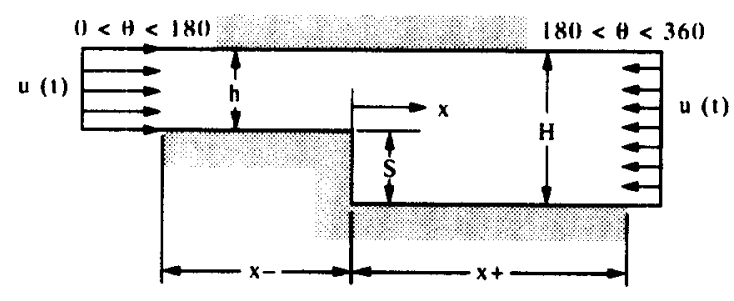

(a) Asymmetric expansion/contraction

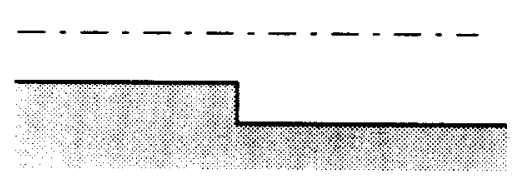

(b) Symmetric expansion/contraction

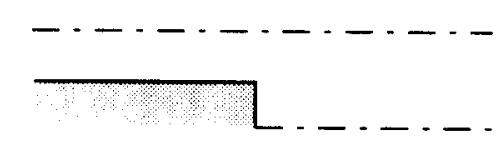

(c) Symmetric blunt body

Fig. 1. The different geometries examined: (a) asymmetric expanaion/contraction; (b) symmetric expansion/contraction; (c) symmetric blunt body. 


\section{Governing Equations}

For unsteady laminar flow the following system of equations is employed.

Continuity equation

$$
\frac{\partial(\rho u)}{\partial x}+\frac{\partial(\rho v)}{\partial y}=0
$$

\section{Momentum equations}

Two equations for momentum result from conservation of momentum in the $x$ - and $y$-directions, respectively:

$x$-momentum,

$$
\frac{\partial(\rho u)}{\partial t}+\frac{\partial\left(\rho u^{2}+\tau_{x x}\right)}{\partial x}+\frac{\partial\left(\rho u v+\tau_{x y}\right)}{\partial y}=-\frac{\partial p}{\partial x}
$$

and

$y$-momentum,

$$
\frac{\partial(\rho v)}{\partial t}+\frac{\partial\left(\rho u v+\tau_{y x}\right)}{\partial x}+\frac{\partial\left(\rho v^{2}+\tau_{y y}\right)}{\partial y}=-\frac{\partial p}{\partial y} .
$$

The momentum equations are cast into a standardized form by utilizing the relationship between the viscous stress and the strain rates:

$$
-\tau_{x y}=-\tau_{y x}=\mu\left(\frac{\partial u}{\partial y}+\frac{\partial v}{\partial x}\right)
$$

Equations of the following form result, which are parabolic in time but elliptic in space coordinates:

$$
\frac{\partial(\rho u)}{\partial t}+\frac{\partial\left(\rho u^{2}-\mu \frac{\partial u}{\partial x}\right)}{\partial x}+\frac{\partial\left(\rho u v-\mu \frac{\partial u}{\partial y}\right)}{\partial y}=-\frac{\partial p}{\partial x}+S_{u}
$$

and

$$
\frac{\partial(\rho u)}{\partial t}+\frac{\partial\left(\rho u v-\mu \frac{\partial v}{\partial x}\right)}{\partial x}+\frac{\partial\left(\rho v^{2}-\mu \frac{\partial v}{\partial y}\right)}{\partial y}=-\frac{\partial p}{\partial y}+S_{v}
$$

where

$$
S_{u}=\frac{\partial\left(\mu \frac{\partial u}{\partial x}\right)}{\partial x}+\frac{\partial\left(\mu \frac{\partial v}{\partial y}\right)}{\partial y}
$$

and

$$
S_{v}=\frac{\partial\left(\mu \frac{\partial u}{\partial y}\right)}{\partial x}+\frac{\partial\left(\mu \frac{\partial v}{\partial x}\right)}{\partial y}
$$

For incompressible/constant property flows, the source terms are zero from the continuity equation.

\section{Dimensionless Parameters}

Different dimensionless parameters characterize the unsteady flow in the channel under consideration:

(1) $\mathrm{Re}_{\max }$; for oscillating flows the mean flow velocity for a cycle is zero, therefore the Reynolds number is based on the maximum amplitude of the velocity during each cycle:

$$
\operatorname{Re}_{\max }=\frac{U_{\max } \cdot D_{\mathrm{h}}}{\frac{\mu}{\rho}}
$$


(2) $\mathrm{Va}$; the frequency of oscillation has been expressed in dimensionless form as the Valensi number:

$$
\mathrm{Va}=\frac{\omega \cdot(\mathrm{d})^{2}}{4.0 \cdot \frac{\mu}{\rho}} .
$$

(3) $A_{r}$; the relative amplitude of fluid displacement is defined as the maximum fluid displacement during half a cycle divided by the channel length, based on the assumption that the fluid moves as a slug flow through the passage:

$$
A_{\mathrm{r}}=\frac{X_{\max }}{L}
$$

Three different physical situations can be identified:

(a) $A_{\mathrm{r}}<1$; part of the fluid oscillates within the passage without exiting.

(b) $A_{\mathrm{r}}=1$; the volume of fluid displaced in half a cycle is exactly equal to the volume of fluid contained within the passage.

(c) $A_{\mathrm{r}}>1$; the volume of fluid displaced during half a cycle is greater than the volume of fluid contained within the passage.

(4) St; the Strouhal number is a combination of $\operatorname{Re}_{\max }$ and Va:

$$
\mathrm{St}=4.0 \cdot\left(\frac{\mathrm{Va}}{\mathrm{Re}_{\max }}\right)
$$

It should be noted that for the same channel geometry, a constant $A_{\mathrm{r}}$ would also imply a constant St.

(5) Another important physical parameter is the channel expansion ratio $h / H$ (sometimes referred to in terms of the step height).

\section{Boundary Conditions}

The following boundary conditions are applied:

(1) Solid walls,

$$
U=V=0
$$

(2) Axis of symmetry,

$$
\frac{\partial u}{\partial y}=0, \quad v=0
$$

(3) Inlet plane

$$
U_{i}=U_{\max } \sin (\omega t) \text {. }
$$

(4) Outlet plane; the exit plane is chosen to be sufficiently far away from the zones of recirculation, therefore the gradients normal to the exit plane (i.e. along the streamwise direction) can be neglected:

$$
\frac{\partial u}{\partial x}=\frac{\partial v}{\partial x}=0
$$

It should be noted that for oscillating flows the inlet and outlet planes are switched at the appropriate time step so that a flow reversal is implemented numerically.

\section{Numerical Method}

The analysis performed utilizes a modified version of the computer code CAST, developed by Peric and Scheuerer [12]. The original code is capable of solving two-dimensional, steady and 
Table 1. The different cases studied in the present work

\begin{tabular}{|c|c|c|c|c|c|c|c|}
\hline $\begin{array}{l}\text { Test } \\
\text { case }\end{array}$ & $\begin{array}{c}\text { Expansion } \\
\text { ratio }\end{array}$ & $\mathbf{R} \mathbf{e}_{\max }$ & $\mathrm{Va}$ & $\begin{array}{c}\text { No. of } \\
\text { axes of } \\
\text { symmetry }\end{array}$ & $A_{\mathrm{r}}$ & $\frac{T_{\mathrm{w}}}{T_{\mathrm{in}}}$ & Mesh \\
\hline $\mathbf{A}$ & $1: 2$ & 187.5 & 1.0 & N/A & 2.0 & 1.2 & $84 \times 22$ \\
\hline $\mathbf{B}$ & $1: 2$ & 187.5 & 1.0 & 1 & 4.0 & 1.2 & $84 \times 22$ \\
\hline C & $1: 2$ & 187.5 & 1.0 & 2 & 4.0 & 1.2 & $84 \times 22$ \\
\hline D & $1: 4$ & 187.5 & 1.0 & $\mathrm{~N} / \mathrm{A}$ & 2.0 & 1.2 & $84 \times 22$ \\
\hline $\mathrm{E}$ & $1: 2$ & 1000.0 & 5.33 & $\mathbf{N} / \mathbf{A}$ & 2.0 & 1.2 & $84 \times 22$ \\
\hline $\mathrm{F}$ & $1: 2$ & 1000.0 & 5.33 & 1 & 4.0 & 1.2 & $84 \times 22$ \\
\hline $\mathrm{G}$ & $1: 2$ & 1000.0 & 5.33 & 2 & 4.0 & 1.2 & $84 \times 22$ \\
\hline $\mathrm{H}$ & $1: 2$ & 1000.0 & 5.33 & 1 & 2.0 & 1.2 & $94 \times 22$ \\
\hline I & $1: 2$ & 1000.0 & 5.33 & 2 & 2.0 & 1.2 & $94 \times 22$ \\
\hline J & $1: 4$ & 1000.0 & 5.33 & $\mathbf{N} / \mathbf{A}$ & 2.0 & 1.2 & $84 \times 32$ \\
\hline $\mathbf{K}$ & $1: 2$ & 2000.0 & 10.66 & $\mathbf{N} / \mathbf{A}$ & 2.0 & 1.2 & $84 \times 22$ \\
\hline L & $1: 4$ & 2000.0 & 10.66 & $\mathbf{N} / \mathbf{A}$ & 2.0 & 1.2 & $84 \times 32$ \\
\hline
\end{tabular}

unsteady, unidirectional flow problems. It has been modified to handle time-dependent boundary conditions for oscillating flows. CAST solves two-dimensional Navier-Stokes equations for laminar flows utilizing a collocated grid. A special velocity-pressure coupling [12] is used, based on the staggered grid concept to prevent oscillatory pressure solution [13]. The numerical solution procedure is a conservative finite volume method using primitive variables such as velocities, pressure and enthalpy. The basic principal involved in this method is to balance the dependent variable fluxes at the inlet and outlet of each control volume within the analysis domain. The solution procedure employed is the well-known SIMPLE algorithm by Patankar [13].

For all cases investigated, the flow cycle was divided into 60 time steps of 6-degree intervals. At least 3 cycles were run for each case to achieve a converged solution. A $0.2 \%$ convergence criteria was used in this study. The CPU time required on a Cray X-MP/Y-MP ranged from 3600 to $8000 \mathrm{~s}$ (for 3 cycles), depending upon the size of the mesh used (see Table 1).

\section{CODE VALIDATION}

Several computational experiments were conducted to validate the CAST code as listed below:

1. The code predictions for the reattachment length and the minimum and maximum velocities at various locations along the channel axis, for $R e=50$ and 150 and expansion ratios of $1: 1.2$ and $1: 1.5$, were compared with similar steady flow results by Morgan et al. [8]; the comparisons were good.

2. Comparisons were made between the present code predictions and the numerical computations by Chiu [6] for steady flow over a backward-facing step, asymmetric channel with a $1: 1.5$ expansion ratio and $\operatorname{Re}=916$. The present code prediction for the friction factor is within $5 \%$ of Chiu's results.

3. Comparison was made, for the size of the separation bubble before the step in a forward-facing step flow at $\operatorname{Re}=200$, between the present code prediction and the numerical computation by Mei and Plotkin [11]. The agreement was within $2 \%$.

4. Also, the present code was used to compute an impulsively started flow over a backward-facing step with $\operatorname{Re}=400$ and a 1:2 expansion ratio. The solution was marched in time and the friction factor and reattachment length were compared with the steady flow results for the same case. The agreement was within $1 \%$. Similar agreement was found upon examining a forward-facing step case.

5. Finally, unsteady flow calculations were conducted for oscillating flow (zero mean) in a straight channel and were in excellent agreement with available analytical solutions for fully developed channel flow [2].

\section{RESULTS AND DISCUSSION}

Table 1 lists the cases studied in this paper. These cases cover wide ranges of $\mathrm{Re}_{\max }$ (from 187.5 to 2000), $\mathrm{Va}$ (from 1 to 10.66$)$, the expansion ratio $(1: 2$ and $1: 4)$ and $A_{\mathrm{r}}(2$ and 4$)$. Also, shown in the table are the three geometric cases discussed above: 


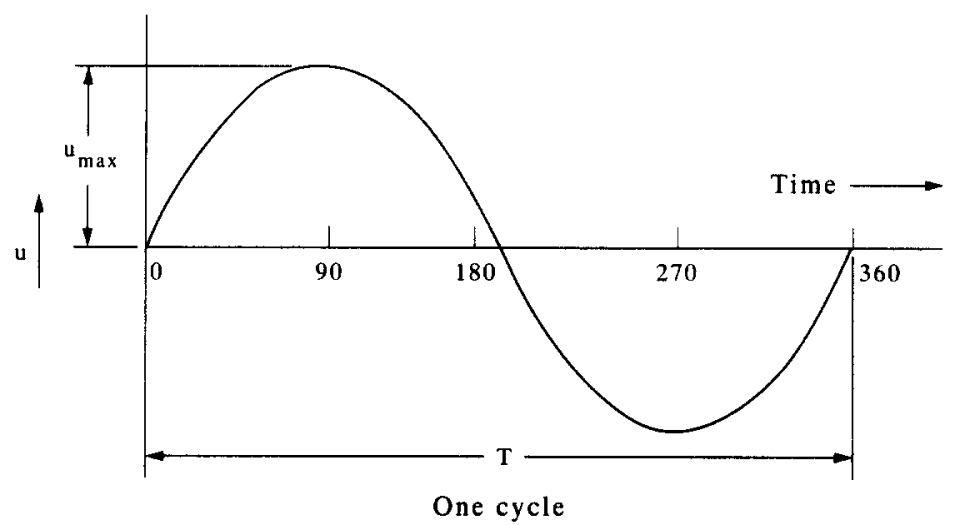

Fig. 2. Time-varying sinusoidal velocity at the channel inlet.

(a) Asymmetric expansion/contraction, cases A, D, E, J, K and L.

(b) Symmetric expansion/contraction, cases B, F and $\mathrm{H}$.

(c) Symmetric blunt body, cases C, G and I.

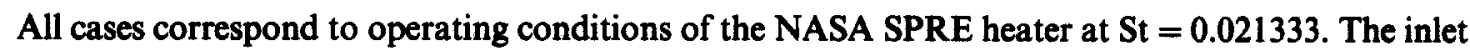
velocity at either end of the channel varies sinusoidally with time, as shown in Fig. 2.

Figure 3 shows the envelope in which different Stirling engines operate, plotted in terms of $\mathbf{R e}_{\max }$ vs Va [4]. In the figure, different criteria $[14,15]$ for the transition from laminar to turbulent flow are shown, for a straight channel. Below these lines (low $\mathbf{R e}_{\max }$ ) the flow will remain laminar throughout the cycle, while above them some combination of laminar/transitional/turbulent flow occurs over the cycle. As evident from the plot, most of the Stirling engine conditions are in the transition or "fully turbulent" zone. Efforts are underway to map the conditions under which quasi-steady turbulence models can be applied to oscillating flow conditions. For more details, see Ref. [16].

The solid circles shown in Fig. 3 are for cases A, E and $\mathbf{K}$ (see Table 1) as well as other runs made (not shown in this paper). We have attempted to identify the region in which the flow is

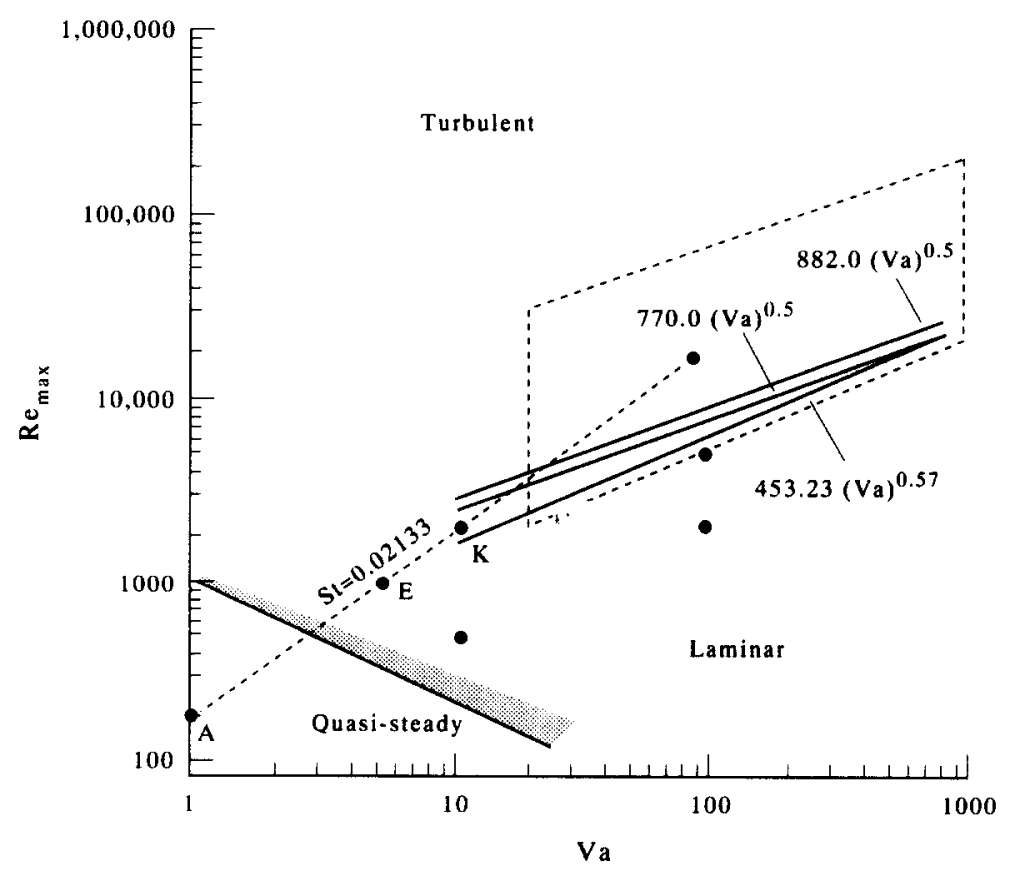

Fig. 3. Envelope in which different Stirling engines operate, together with: (i) the criterion for transition from laminar to turbulent flow in straight channels, $R_{e_{\max }}=822 \times\left(\mathrm{Va}^{0.5}\right), \mathbf{R e}_{\max }=770 \times\left(\mathrm{Va}^{0.5}\right)$ and $\mathrm{Re}_{\max }=453.23 \times\left(\mathrm{Va}^{0.57}\right)$; (ii) the different cases studied in the present work. 
laminar quasi-steady. Case $\mathrm{A}$ lies in that region, while cases $\mathrm{E}$ and $\mathrm{K}$ are in the non-quasi-steady region (to be discussed in more detail later). The shaded area in the figure indicates our estimation (outcome of the present analysis) that below it the flow is laminar quasi-steady, while above it the flow becomes laminar non-quasi-steady.

\section{Effect of the Geometry}

Figure 4 shows the streamlines of the oscillating flow at different velocity phase angles of 30 , $60,90,120,150,180,210,240,270,300,330$ and 360 . The results are for $\operatorname{Re}_{\max }=187.5, \mathrm{Va}=1$ and an asymmetric expansion/contraction with a ratio of $1: 2$ (case A). Figure 5 shows similar results for a symmetric expansion/contraction (case B); while Fig. 6 is for a symmetric blunt body (case $\mathrm{C}$ ). From the plots it can be seen that in the sudden expansion process, the separation bubble behind the step grows gradually during flow acceleration and then shrinks gradually during flow

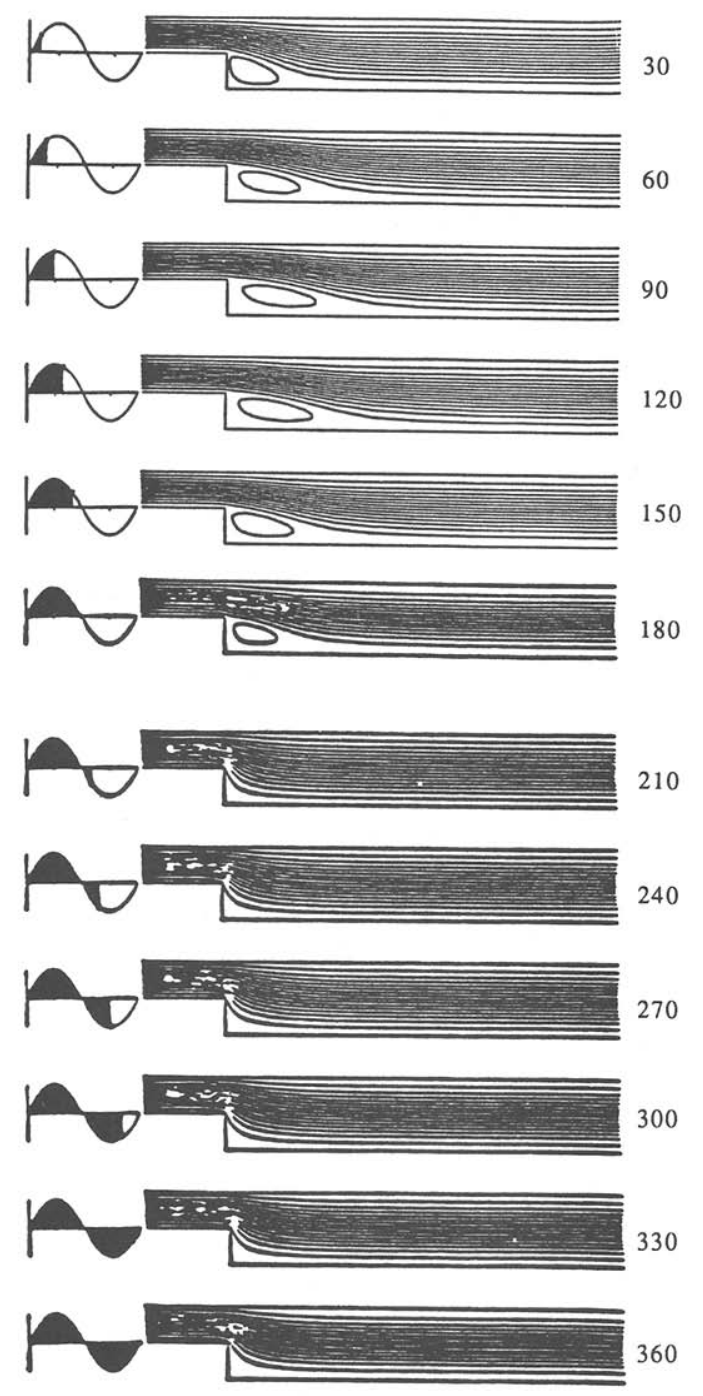

Case A

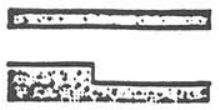

Fig. 4. Streamlines of oscillating flow at different velocity phase angles, for $\operatorname{Re}_{\max }=187.5$ and $\mathrm{Va}=1$. (Case $\mathrm{A}$, asymmetric expansion/contraction with a ratio of $1: 2$.)
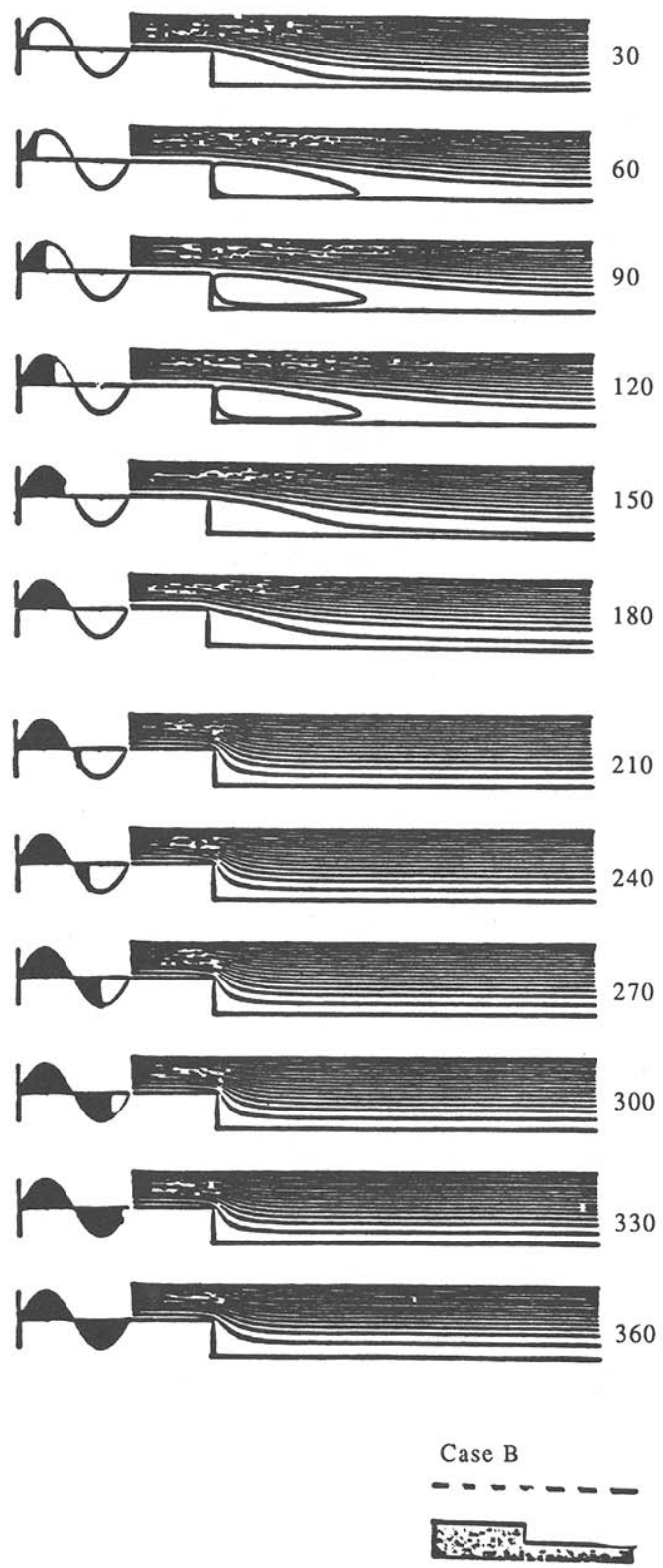

Fig. 5. Streamlines of oscillating flow at different velocity phase angles, for $\operatorname{Re}_{\max }=187.5$ and $\mathrm{Va}=1$. (Case $\mathrm{B}$, symmetric expansion/contraction with a ratio of $1: 2$.) 
deceleration. However, this bubble disappears completely during the flow reversal (sudden contraction). Also, as expected, the size of the separation bubble (at a given velocity phase angle) gets progressively bigger from $A$ to $B$ to $C$. These cases, as described above, show a quasi-steady behavior.

As for the friction factor results (not shown in this paper for all cases); it was found that for case $B$ the friction factor is of the same order of magnitude as the asymmetric expansion (case A). The difference lies in the developing zone beyond the reattachment point. Similar trends are observed for $\mathrm{Re}_{\max }=1000$ - cases $\mathrm{E}, \mathrm{F}$ and $\mathrm{G}$.

\section{Effect of $\operatorname{Re}_{\text {max }}$ and $\mathrm{Va}$}

Figure 7 shows the streamlines of the oscillating flow at different velocity phase angles of 30 , $60,90,120,150,180,210,240,270,300,330$ and 360. The results are for $\operatorname{Re}_{\max }=500, \mathrm{Va}=10.66$ and an asymmetric expansion/contraction with a ratio of $1: 2$. Similar results are shown in Fig. 8 for $\operatorname{Re}_{\max }=1000, \mathrm{Va}=10.66$ and an asymmetric expansion/contraction with a ratio of $1: 2$ (case

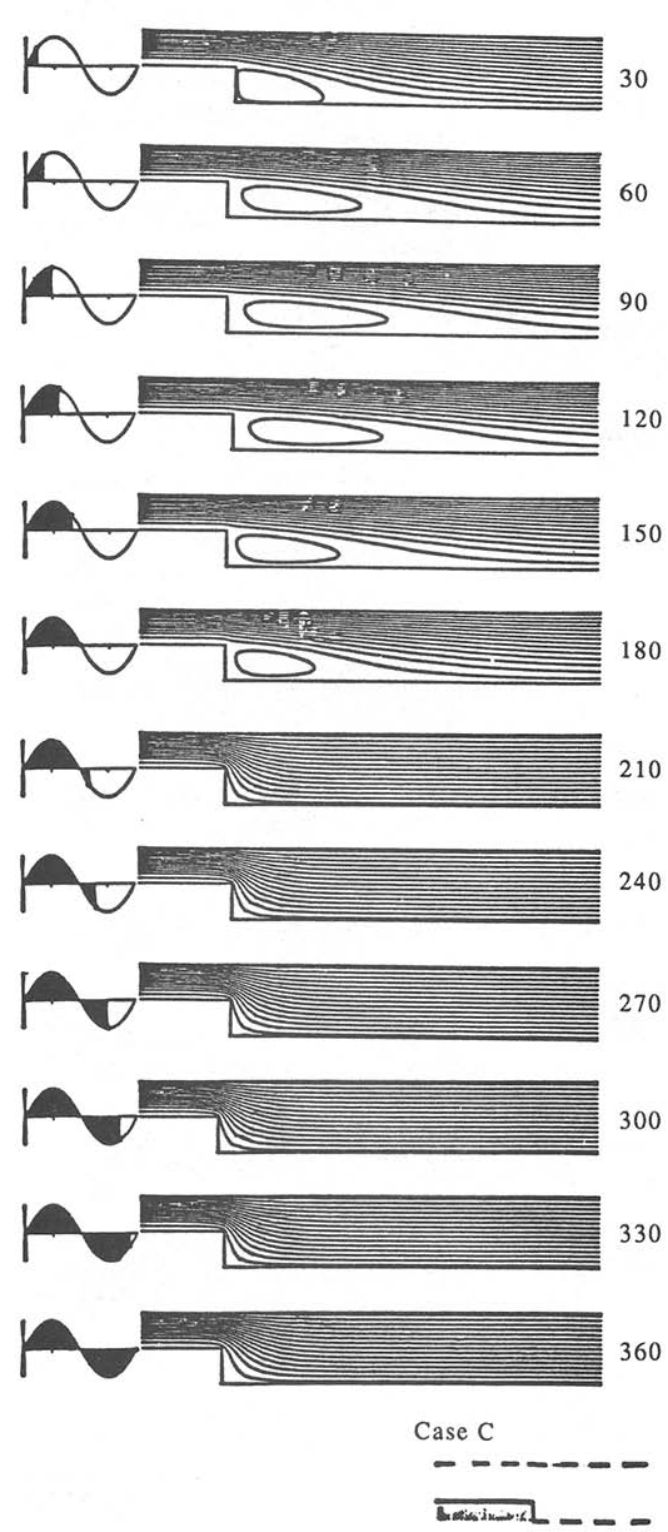

Fig. 6. Streamlines of oscillating flow at different velocity phase angles, for $\operatorname{Re}_{\max }=187.5$ and $\mathrm{Va}=1$. (Case $\mathrm{C}$, symmetric blunt body with a ratio of $1: 2$.)

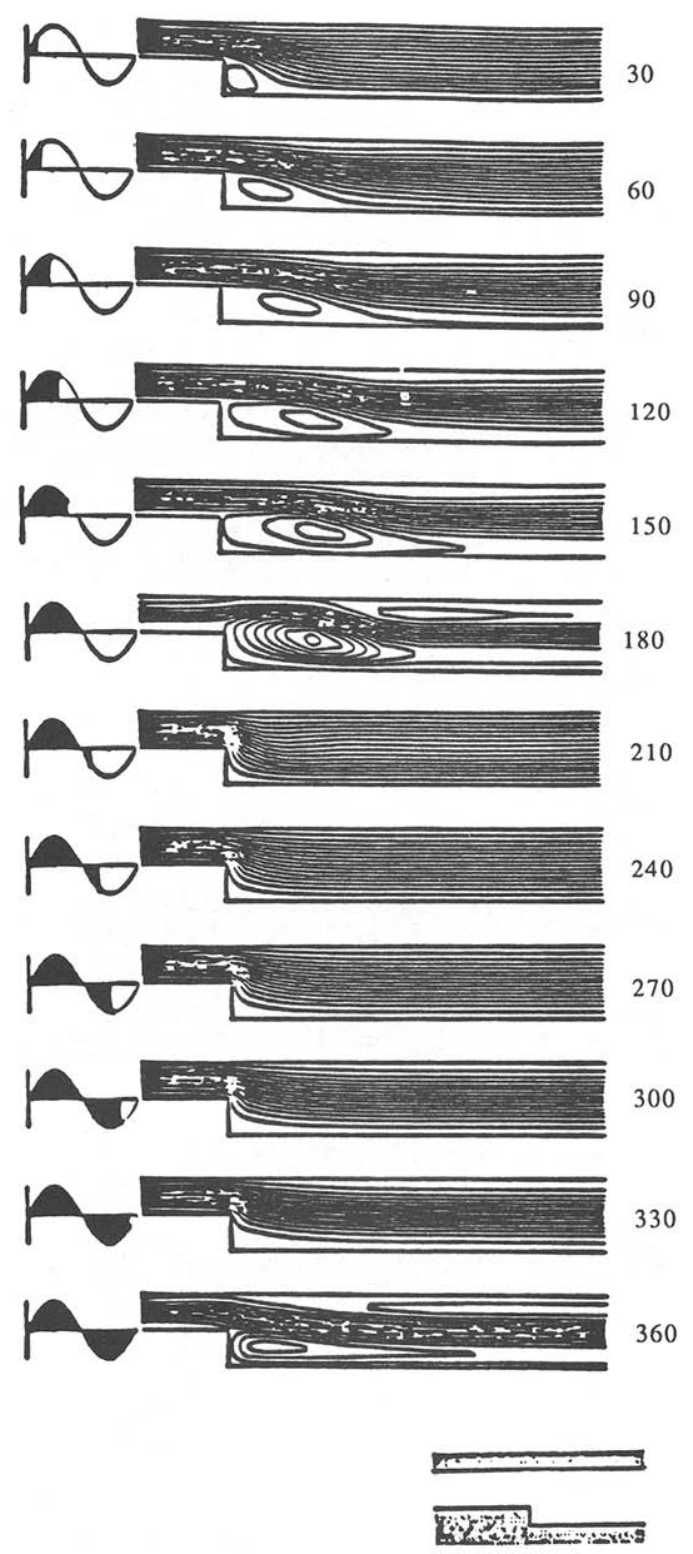

Fig. 7. Streamlines of oscillating flow at different velocity phase angles, for $\mathrm{Re}_{\max }=500$ and $\mathrm{Va}=10.66$ (Asymmetric expansion/contraction with a ratio of $1: 2$.) 
E). Experimental and analytical results for a straight channel[4], also see Fig. 3, indicate that the flow can be assumed laminar for the two cases. Due to the lack of experimental evidence for the channel with a sudden change in cross section, the flow is considered laminar. It was observed while watching the animation videos of the above cases that as the fluid accelerates during the sudden expansion phase, the separation bubble region grows in magnitude and physical size. This effect is observed irrespective of the magnitude of the flow Re. However, during the flow deceleration, this separation bubble loses momentum rapidly and disappears for cases A-D (low Re) but continues to grow while losing momentum for cases E-L (relatively higher Re). This observation, can be seen also by comparing Figs 4,7 and 8 .

For the cases presented, $\mathrm{Va}$ is also increased with the Re to maintain a constant St corresponding to the actual Stirling engine.

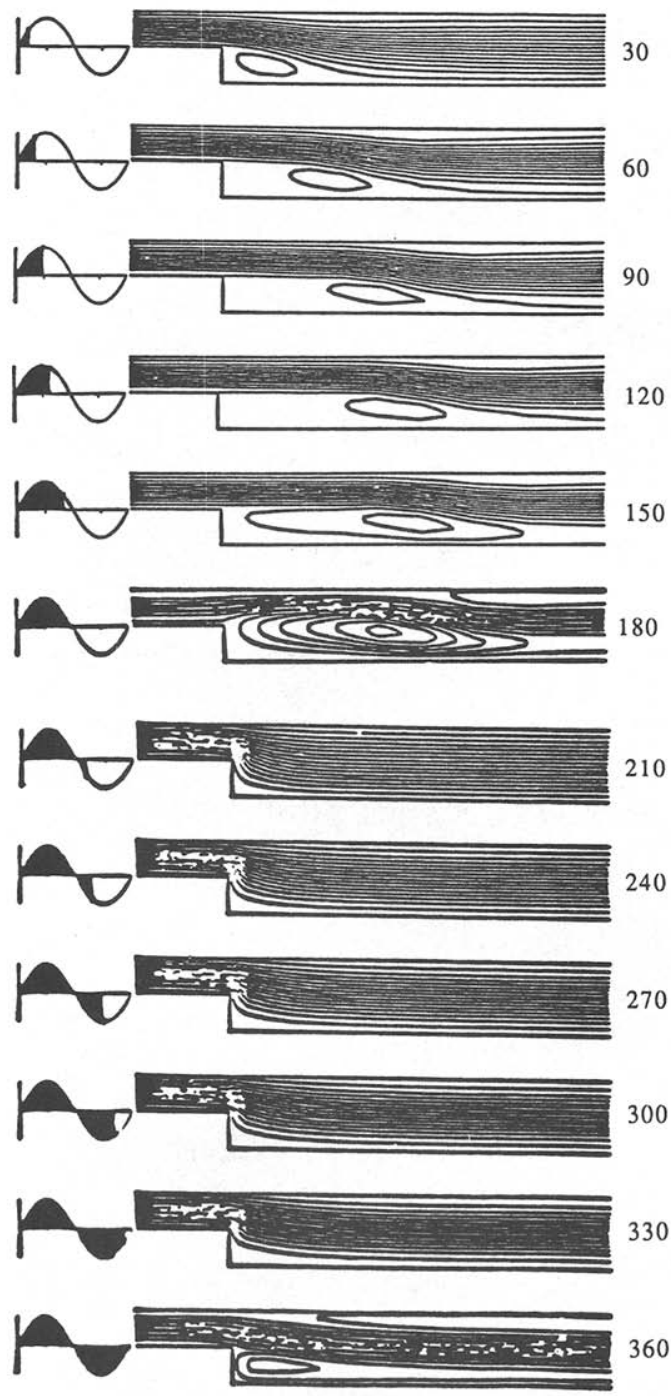

Case E

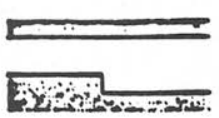

Fig. 8. Streamlines of oscillating flow at different velocity phase angles, for $R e_{\max }=1000$ and $\mathrm{Va}=5.33$ (Case $\mathrm{E}$, asymmetric expansion/contraction with a ratio of $1: 2$.)

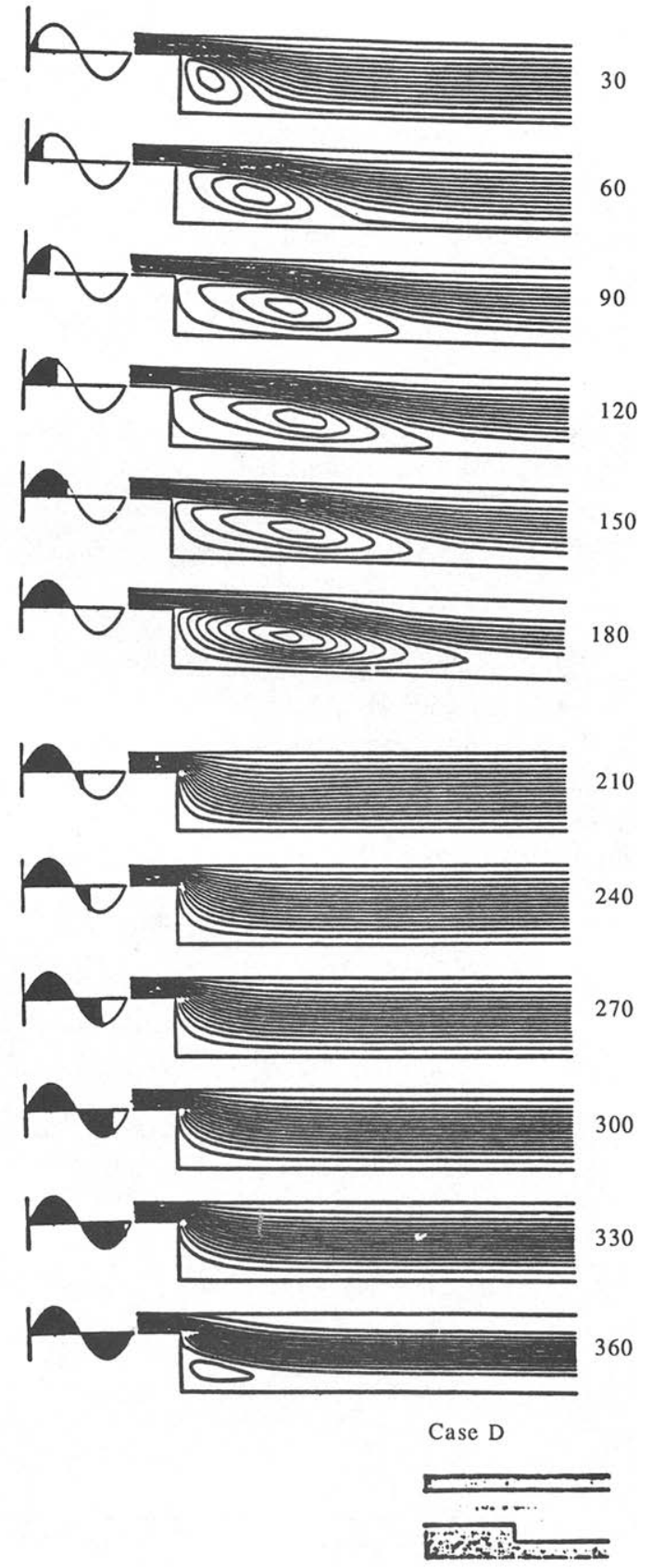

Fig. 9. Streamlines of oscillating flow at different velocity phase angles, for $\operatorname{Re}_{\max }=187.5$ and $\mathrm{Va}=1$. (Case $\mathrm{D}$, asymmetric expansion/contraction with a ratio of $1: 4$.) 


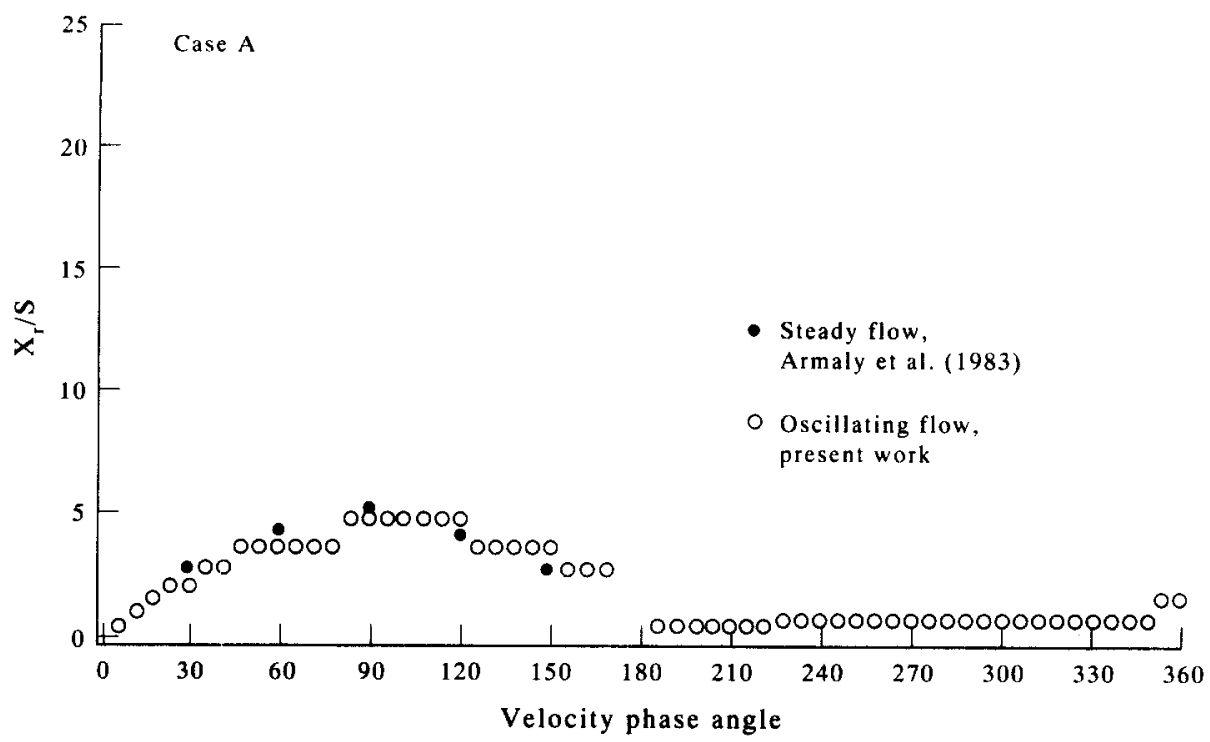

Fig. 10. Reattachment length vs time for oscillating flow at $\operatorname{Re}_{\max }=187.5$ and $\mathrm{Va}=1$. (Case $\mathrm{A}$, asymmetric expansion/contraction with a ratio of $1: 2$.)

It was found from the analysis in this work that as Va increases, the flow becomes nonquasi-steady at a lower $\mathbf{R e}_{\max }$. This is shown in Fig. 3 by the shaded area, below which the flow can be considered quasi-steady. Also, this could lead to the conclusion that as Va increases the flow is likely to become turbulent at a much lower $\operatorname{Re}_{\max }$ than normally observed for straight channels.

\section{Effect of the Expansion Ratio}

Figure 9 shows the streamlines of the oscillating flow at different velocity phase angles of 30 , $60,90,120,150,180,210,240,270,300,330$ and 360 . The results are for $\operatorname{Re}_{\max }=187.5, \mathrm{Va}=1$ and an asymmetric expansion/contraction with a ratio of $1: 4$ (case D).

Comparing cases $A$ and $D$ (both are similar except for the expansion ratio), the recirculation zone in case $\mathrm{D}$ grows to almost 4 times the size in case $A$. The recirculation zone, being larger, dissipates its energy during the fluid deceleration and experiences some growth. This affects the

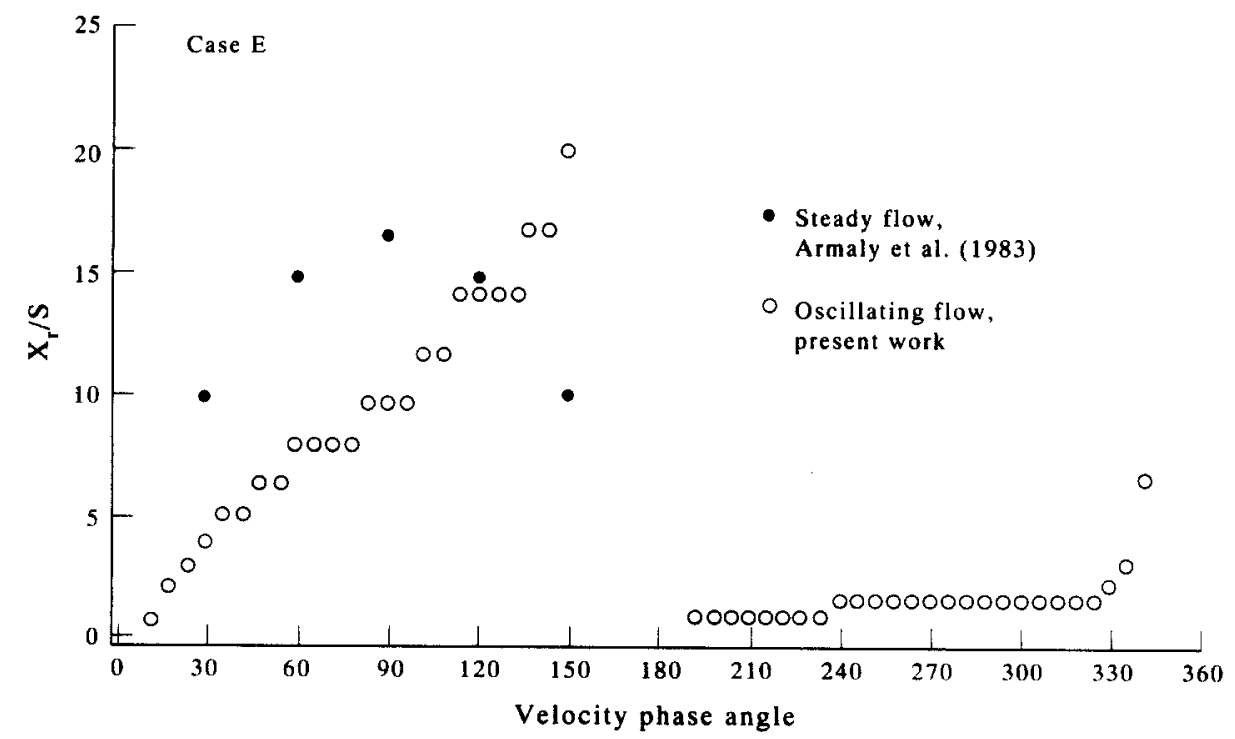

Fig. 11. Reattachment length vs time for oscillating flow at $\operatorname{Re}_{\max }=1000$ and $\mathrm{Va}=5.33$. (Case $\mathrm{E}$, asymmetric expansion/contraction with a ratio of $1: 2$.) 
friction factor by more than an order of magnitude (not shown in this paper). Also, because of a larger recirculation zone, which results in good mixing, wall heat flux is reduced (not shown in this paper). Again, by comparing cases A and D, it can be seen that although case A is quasi-steady, case $D$ is not because of the higher expansion ratio.

For a higher $R e$, cases $E$ and $J\left(R e_{\max }=1000\right)$ and cases $K$ and $L\left(\operatorname{Re}_{\max }=2000\right)$, a similar trend regarding the size of the recirculation zone and the corresponding effect on friction and heat transfer is observed.

\section{Reattachment Length}

Figure 10 shows the reattachment length vs velocity phase angle for $\operatorname{Re}_{\max }=187.5, \mathrm{Va}=1$ and an asymmetric expansion/contraction with a ratio of $1: 2$ (case A). Also, shown is the reattachment length, given by Armaly et al. [5], for steady flow at the instantaneous Re. The results of the present analysis show good agreement, indicating that the flow is quasi-steady as explained earlier. On the other hand, Fig. 11 shows a similar plot of the reattachment length vs velocity phase angle at a higher $\operatorname{Re}_{\max }=1000, \mathrm{Va}=5.33$ (case $\mathrm{E}$ ). It can be seen from Fig. 11 that the reattachment length will grow initially at a lower rate than in the corresponding quasi-steady case, thereafter it will

(a)

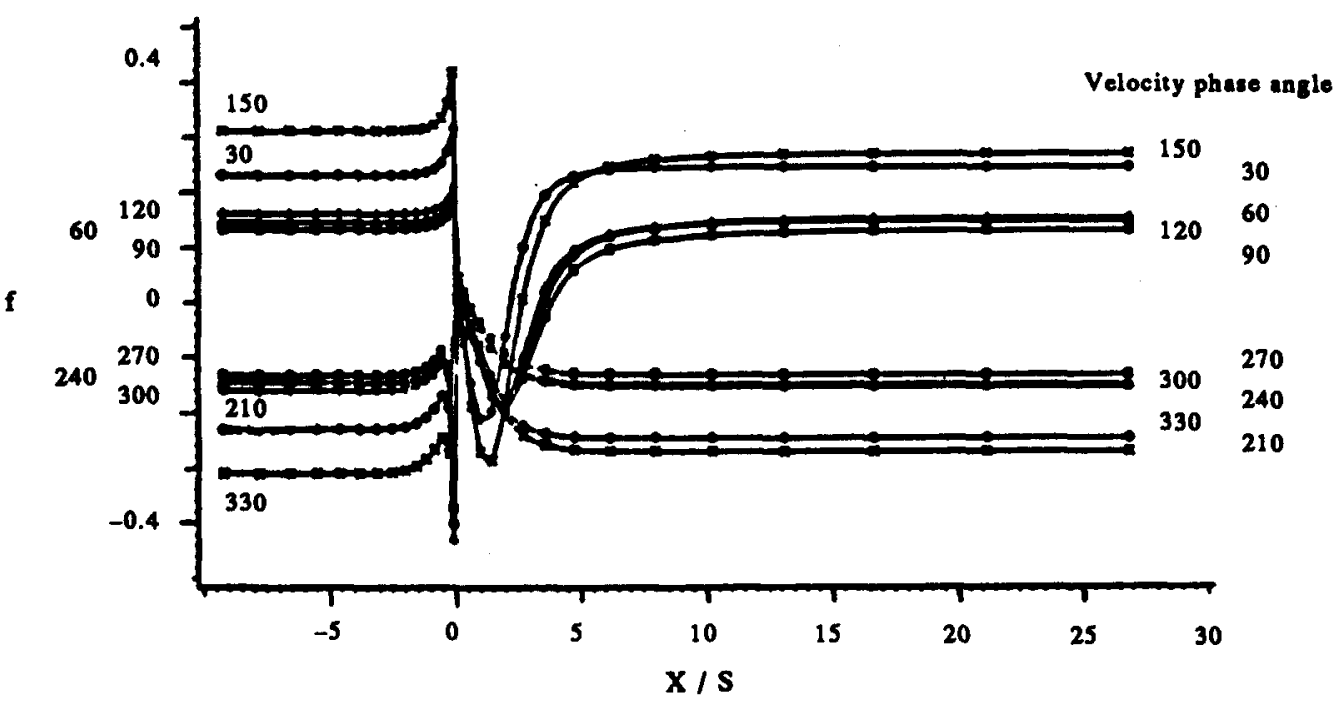

(b)

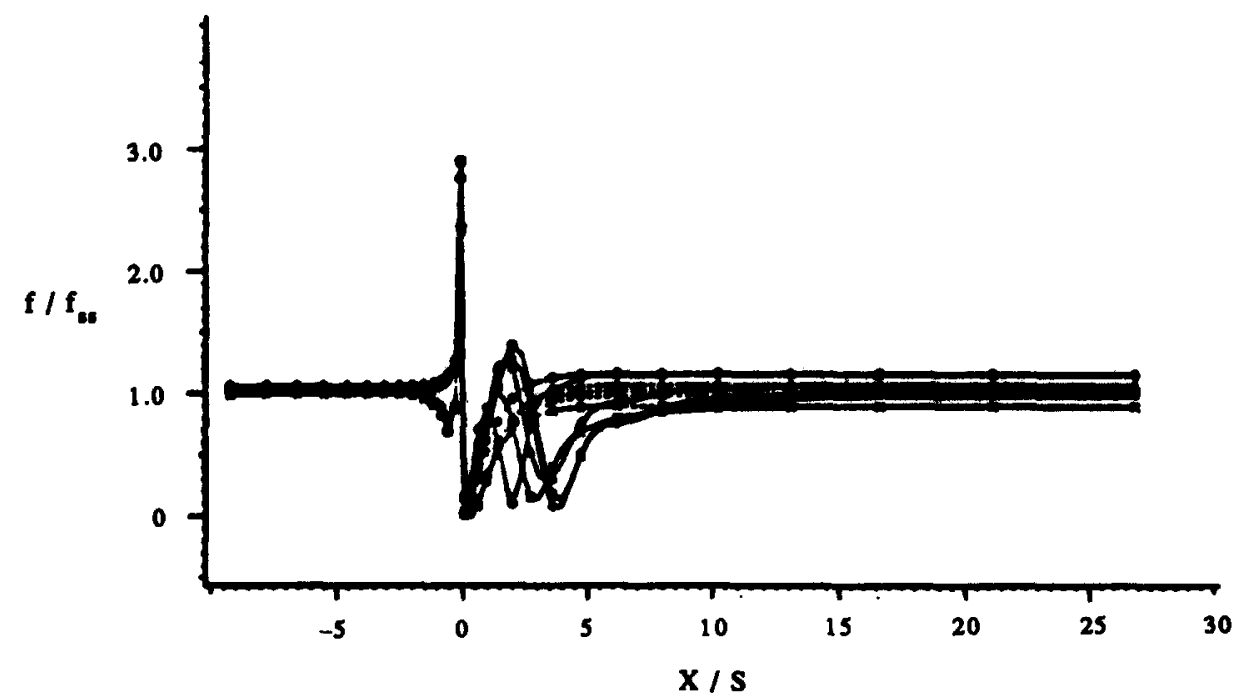

Fig. 12. (a) Friction factor vs time for oscillating flow at $R_{e_{\max }}=187.5$ and $\mathrm{Va}=1$. (Case $\mathrm{A}$, asymmetric expansion/contraction with a ratio of $1: 2$.). (b) Normalized friction factor vs time for oscillating flow at

$R e_{\max }=187.5$ and $\mathrm{Va}=1$. (Case $A$, asymmetric expansion/contraction with a ratio of $1: 2$.) 
continue to grow at a higher rate and then disappear during flow reversal, i.e. case $E$ is non-quasi-steady.

\section{Friction Factor}

Figure 12(a) shows the instantaneous friction factor vs dimensionless axial distance at different velocity phase angles, for $\mathrm{Re}_{\max }=187.5, \mathrm{Va}=1$ and an asymmetric expansion/contraction with a ratio of 1:2 (case A). A similar plot is shown in Fig. 12(b) but with the coordinate being the instantaneous friction factor divided by the steady-state friction factor at the instantaneous Re. The quasi-steady behavior is observed by having values of $f / f_{\mathrm{ss}}$ close to $1.0 \mathrm{in}$ Fig. 12(b) at all times and most of the channel axial locations.

Figure 13(a) shows the instantaneous friction factor vs dimensionless axial distance at different velocity phase angles, for $\mathrm{Re}_{\max }=1000, \mathrm{Va}=5.33$ and an asymmetric expansion/contraction with a ratio of $1: 2$ (case E). A similar plot is shown in Fig. 13(b) but with the coordinate being the instantaneous friction factor divided by the steady-state friction factor at the instantaneous Re. It can be seen from Fig. 13(b) that $f / f_{\text {ss }}$ departs considerably from 1.0, particularly after the step, indicating a non-quasi-steady behavior. The friction factor can be a factor of 2 higher or lower than the steady flow values for $X / S$ east from the reattachment location. This indicates

(a)

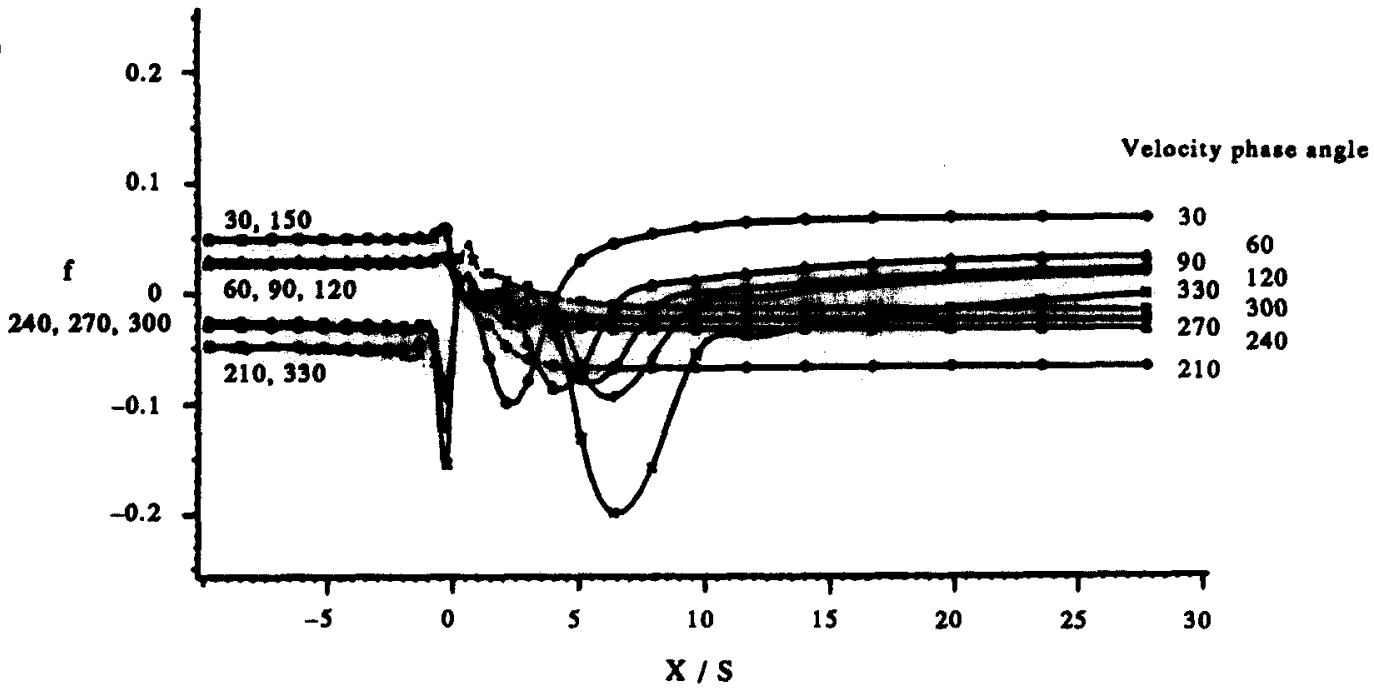

(b)

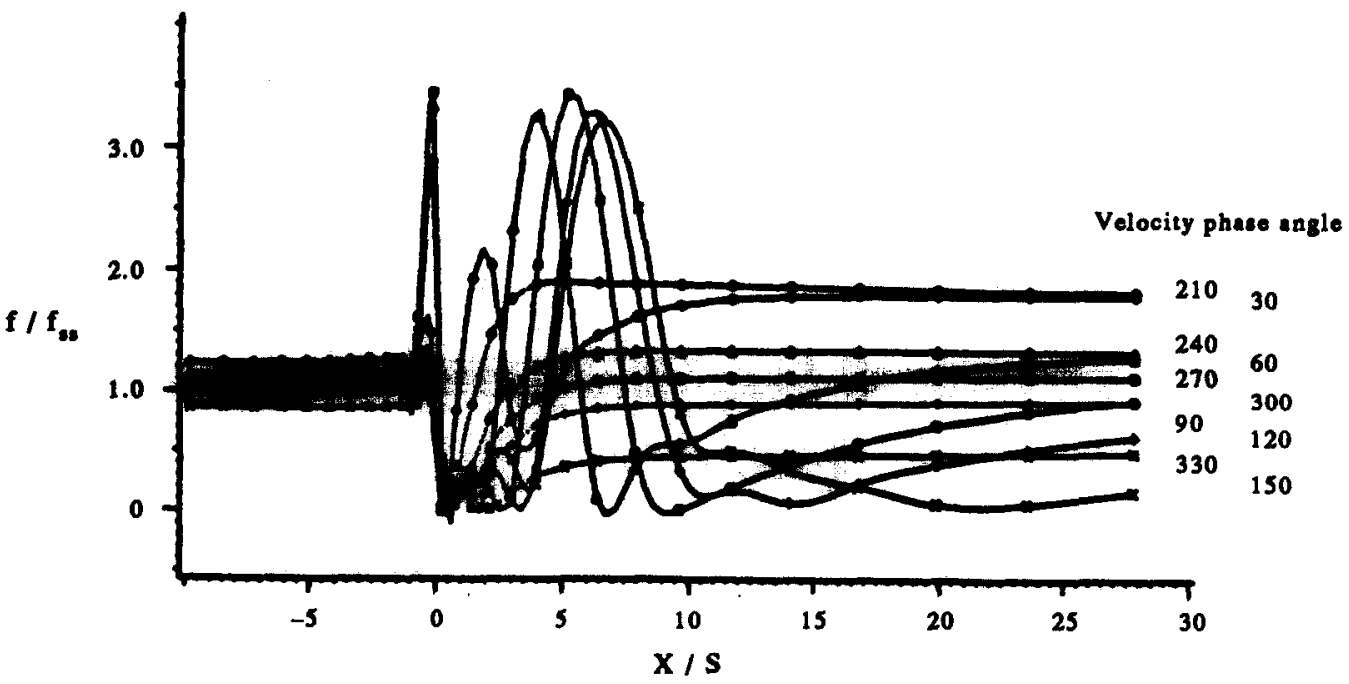

Fig. 13. (a) Friction factor vs time for oscillating flow at $R_{\max }=1000$ and $\mathrm{Va}=5.33$. (Case $\mathrm{E}$, asymmetric expansion/contraction with a ratio of $1: 2$.). (b) Normalized friction factor vs time for oscillating flow at

$\mathrm{Re}_{\max }=1000$ and $\mathrm{Va}=5.33$. (Case $\mathrm{E}$, asymmetric expansion/contraction with a ratio of $1: 2$.) 
that the steady-state friction factor correlations cannot be used in such applications. Work is underway to develop new correlations for the friction factor and pressure drops for oscillating flow conditions.

\section{CONCLUDING REMARKS}

In this paper the oscillating flow (zero mean) between two parallel plates with a sudden change in cross section was studied. The flow was assumed to be laminar incompressible with the inflow velocity uniform over the channel cross section but varying sinusoidally with time. Under these conditions, the fluid undergoes a sudden expansion in one-half of the cycle and a sudden contraction in the other half. The flow reversal, under such conditions, is caused not only by the flow oscillations but also by the sudden change in cross section.

A computer code, CAST, developed by Peric and Scheuerer [12] has been modified to handle time-varying boundary conditions. The CAST code solves Navier-Stokes equations in 2-D using a finite volume method. The code has been validated by comparing its predictions with available computational, experimental and analytical data for straight channels and those with a sudden change in cross section. Good agreements were found for the friction factor, reattachment length and minimum and maximum velocities at different axial channel locations.

The computations were extended to oscillating flow conditions. The cases examined emulate the operating parameters of the NASA SPRE (Space Power Research Engine). The $\mathbf{R e}_{\max }$ in cases presented here is chosen to be sufficiently low that the assumption of laminar flow holds true during the entire flow cycle. The cases examined are summarized in Table 1.

In all cases examined, a separation zone appears during the sudden expansion and grows as the flow accelerates. This growth, however, depends on the $\operatorname{Re}_{\max }$. For low $\operatorname{Re}_{\max }(=187.5)$, the growth follows a quasi-steady behavior, while for the higher $\operatorname{Re}_{\max }(=1000)$ the growth is very rapid and the separation bubble continues growing during flow deceleration (non-quasi-steady behavior).

A shaded area has been identified (see Fig. 3), below which the flow is laminar quasi-steady. This indicated that as $\mathrm{Va}$ increases the flow becomes non-quasi-steady at a lower $\mathbf{R e}_{\max }$.

When the flow reverses, the fluid goes through a sudden contraction and the recirculation bubble from the previous half-cycle is swept back into the smaller section of the channel.

Such flow behavior causes the instantaneous friction factor to deviate substantially from the steady-state friction factor for the same flow parameters. The friction factor can be a factor of 2 higher or lower than the steady flow values for $X / S$ east from the reattachment location. Work is underway to develop new correlations for the friction factor and pressure drops under oscillating flow conditions.

Upon examining the effect of the channel expansion ratio, it was found that for the same $\operatorname{Re}$ and $\mathrm{Va}$, increasing the expansion ratio increases the friction losses. This is consistent with the observations made by several researchers studying steady flows with a change in the channel cross section.

Acknowledgements - This research has been conducted under NASA grant No. NAG3-971. Mr Roy C. Tew Jr, of NASA Lewis Research Center, is greatly acknowledged for his encouragement and helpful suggestions.

\section{REFERENCES}

1. M. B. Ibrahim, R. C. Tew and J. E. Dudenhoefer, Two-dimensional numerical simulation of a Stirling engine heat exchanger. NASA Technical Memorandum 102057 (1989).

2. U. H. Kurzweg, Enhanced heat conduction in oscillating viscous flows within parallel-plate channels. J. Fluid Mech. 156, 291 (1985).

3. T. W. Simon and J. R. A. Seume, Survey of oscillating flow in Stirling engine heat exchangers. NASA Contractor Report 182108 (Mar. 1988).

4. J. R. Seume, An experimental investigation of transition in oscillating pipe flow. Ph.D. Thesis, Univ. of Minnesota, Minneapolis, MN (1988).

5. B. F. Armaly, F. Durst, J. C. F. Pereira and B. Schonung, Experimental and theroetical investigation of backward-facing step flow. J. Fluid Mech. 127, 473 (1983).

6. I. T. Chiu, Prediction of laminar flows over rearward-facing step using the partially-parabolized Navier-Stokes equations. Master's Thesis, Iowa State Univ., Ames, IA (1984).

7. O. K. Kwon, R. H. Pletcher and J. P. Lewis, Prediction of sudden expansion flows using the boundary-layer equations. J. Fluids Engng 106, 285 (1984).

8. K. Morgan, J. Periaux and F. Thomasset, Analysis of laminar flow over a backward-facing step. In Proceedings of $a$ GAMM Workshop. Vieweg, Braunschweig (1984). 
9. A. Baron, F. K. Tsou and W. Aung, Flow field and heat transfer associated with laminar flow over a forward-facing step. In Heat Transfer 1986: Proc. 8th Int. Conf., San Francisco, CA, Vol. 3, pp. 1077-1082 (1986)

10. S. Hafez and M. A. Serag-Eldin, Computation of the recirculating flow over and under bluff-bodies. In Proc. 4th Int. Conf. on Numerical Methods in Laminar and Turbulent Flows, Vol. 1, Chap. 1, pp. 687-695 (1985).

11. R. W. Mei and A. Plotkin, Navier-Stokes solutions for laminar incompressible flows in forward-facing step geometries. AIAA Jl 24, (1986).

12. M. Peric and G. Scheuerer, CAST-a finite volume method for predicting two-dimensional flow and heat transfer phenomena. GRS-Technische Notiz SSR-89-01 (Sept. 1989).

13. S. V. Patankar, Numerical Heat Transfer and Fluid Flow. Hemisphere, Washington, DC (1980).

14. M. Ohmi and M. Iguchi, Critical Reynolds number in an oscillating pipe flow. Bull. JSME 25, 165 (1982).

15. M. Hino, M. Sawamoto and S. Takasu, Experiments on the transition to turbulence in an oscillatory pipe flow. J. Fluid Mech. 131, 363 (1983).

16. K.-H. Ahn and M. B. Ibrahim, A 2-D oscillating flow analysis in Stirling engine heat exchangers. NASA Technical Memorandum 103781 (1991). 